\title{
Observation of plasma wakefield acceleration in the underdense regime
}

\author{
N. Barov and J. B. Rosenzweig \\ Department of Physics and Astronomy, UCLA, 405 Hilgard Avenue, Los Angeles, California 90095-1547 \\ M. E. Conde, W. Gai, and J. G. Power \\ High Energy Physics Division, Argonne National Laboratory, 9700 S. Cass Avenue, Argonne, Illinois 60439
}

(Received 22 July 1999; published 13 January 2000)

\begin{abstract}
Initial experiments which have explored the physics of the underdense (blowout) regime of the plasma wakefield accelerator (PWFA) at the Argonne Wakefield Accelerator facility are reported. In this regime, the relativistic electron beam is denser than the plasma, causing the beam channel to completely rarefy, and leaving a high quality accelerating region which also contains a uniform ion column. This ion column in turn allows the drive and accelerating beams to be well guided over many initial beam beta-function lengths. The results of these experiments, which have taken place over several years, are reviewed. Notable achievements in the course of these studies include the creation and measurement of drive and witness beam generated in an rf photoinjector, as well as previously published studies on drive beam guiding in the underdense regime. In addition, these experiments allowed measurement of both beam energy loss and gain, at a maximum average rate of $25 \mathrm{MeV} / \mathrm{m}$ in this regime of the PWFA, which is consistent with a peak acceleration gradient of $62 \mathrm{MeV} / \mathrm{m}$ in the excited waves. Difficulties associated with this type of experiment are discussed, as are prospects for mitigating these difficulties and achieving high gradient acceleration in planned future experiments.
\end{abstract}

PACS numbers: 52.40.Mj, 52.75.Di, 29.17.+w, 29.27.-a

\section{INTRODUCTION}

Much progress has been made in recent years in the experimental demonstration of acceleration in plasmas. The basic mechanisms for excitation of electron plasma waves which support accelerating fields has been verified, and accelerating gradients in excess of $30 \mathrm{GeV} / \mathrm{m}$ have been observed [1,2]. Despite this progress, however, many problems concerning preservation of the beam quality during acceleration in high gradient plasma waves remain experimentally unaddressed; plasma wave fields tend to be nonuniform in their accelerating fields, and nonlinear in transverse focusing fields. It is critical, from the point of view of application of plasma acceleration to high energy physics, that these concerns be mitigated.

Operation of the plasma wakefield accelerator (PWFA) [3] in the extremely nonlinear ("blowout," where the beam is denser than the plasma) regime was originally proposed by Rosenzweig, Breizman, Katsouleas, and Su in 1991 [4]. While this system is nonlinear from the point of view of the plasma response-all of the plasma electrons are driven out of the beam channel by the intense fields of the driving beam-the attributes of the accelerating and focusing fields are what accelerator physicists commonly refer to as linear. Development of this regime represents a serious attempt at formulating a version of a plasma accelerator which has the attributes of a standard rf linear accelerator [5]. These attributes include: (i) focusing which, for electrons, is linear in offset from the symmetry axis, and independent of longitudinal position within the wave; (ii) acceleration which is dependent only on longitudinal position within the accelerating wave, and not on transverse offset; and (iii) multi-GeV gradient operation at $\mathrm{mm}$ wavelengths. The physical reasons for these attributes are displayed in part by Fig. 1, and are discussed further here.

The beam, which has peak density $n_{b}$ well in excess of the ambient plasma electron density $n_{0}$, ejects all of the plasma electrons from its propagation channel before the bulk of the beam has passed. Behind this point inside of the plasma electron-rarefied region, the fields are a superposition of an electrostatic component due to the nearly stationary ions and a relativistic phase velocity traveling electromagnetic wave. The ion-induced fields provide electron beam particle focusing which is

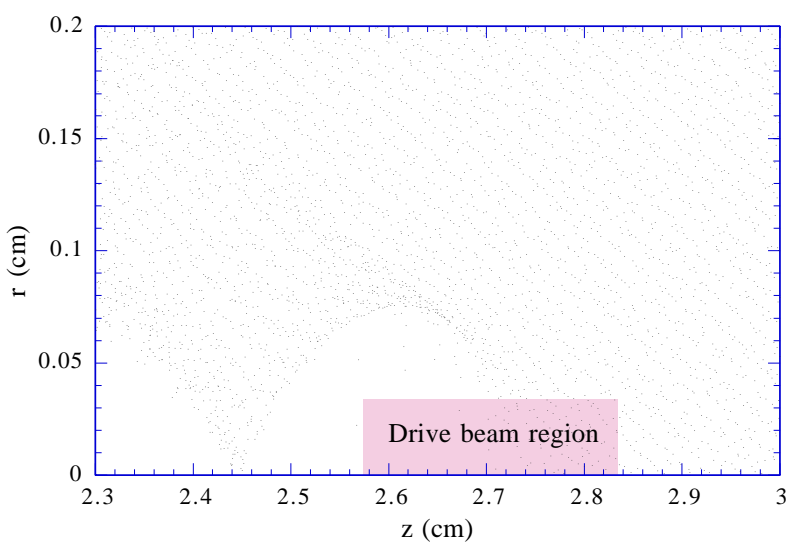

FIG. 1. (Color) Particle-in-cell code (Barov [6]) simulation of blowout regime, with beam density much larger than plasma density. 
dependent only on the plasma ion charge density $+e n_{0}$,

$$
F_{r}=-e E_{r}=-2 \pi e^{2} n_{0} r \equiv-\frac{1}{2} m_{e} \nu_{b}^{2} k_{p}^{2} r,
$$

where $k_{p}^{2}=\omega_{p}^{2} / \nu_{b}^{2}$. This force thus allows simple, uniform, and linear focusing of the portion of the drive beam inside of the rarefied region, as well as the beam which accelerates near the back of the rarefied region. The focusing fields arise purely from the ions; the net transverse force due to axisymmetric electromagnetic (TM) $E_{r}$ and $H_{\phi}$ fields cancel in the ultrarelativistic limit.

Acceleration inside of the rarefied channel displays a nonsinusoidal dependence on longitudinal position $(\xi=$ $z-\nu_{b} t$ ) within the accelerating wave, typically a sawtoothlike electric field profile [3] which rises steeply as the very high plasma electron density region $(z \cong 2.44 \mathrm{~cm}$ in Fig. 1) is approached. This density spike is formed when many of the plasma electrons which are blown out return to the axis and cross it in a nonlaminar manner. Note that even though laminarity is violated (the wave is broken), a new rarefied region exists behind the density spike, and the nonlinear wave pattern can continue beyond the first half-oscillation of the plasma electrons.

As the axisymmetric, TM electromagnetic wave which travels with plasma disturbance has an ultrarelativistic phase velocity, not only does its net transverse force cancel [to order $\gamma_{b}^{-2}=1-\left(\nu_{b} / c\right)^{2}$ ], the acceleration is nearly independent of radial offset in the rarefaction region, as shown in the fluid simulation [7] results displayed in Fig. 2.

It is interesting to note that in the simulations shown in Figs. 1 and 2, the fields approach or exceed the nonrelativistic plasma wave-breaking limit [8-12], $e E_{W B}=$ $m_{e} c^{2} k_{p} \cong \sqrt{n_{0}\left(\mathrm{~cm}^{-3}\right)}(\mathrm{eV} / \mathrm{cm})$. This allows for high gradient operation at longer wavelengths, due to lower (relative to the linear regime) plasma densities and relativistic lengthening of the plasma oscillation period. The experiments described here operate in the range $n_{0} \approx$ $10^{13} \mathrm{~cm}^{-3}$, with experiments $[4,13,14]$ either planned or

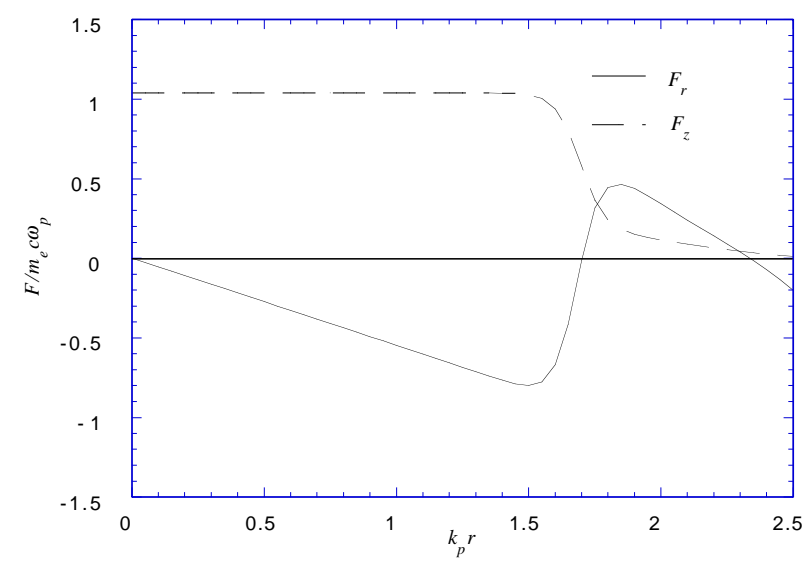

FIG. 2. (Color) Wakefield forces in acceleration phase of rarefied plasma wave, as a function of radius, from fluid code NOVO (Breizman et al. [7]). already begun designed to run at densities in excess of $n_{0} \approx 10^{14} \mathrm{~cm}^{-3}$, this implies possible accelerating gradients around $1 \mathrm{GeV} / \mathrm{m}$ for presently used plasmas. The relatively long wavelength is an advantage for beam dynamics, as the injected beam parameters are relaxed [15], and the smaller plasma density mitigates transverse emittance growth due to multiple scattering of the beam off of plasma ions $[4,16]$.

While the strength of the acceleration in the underdense (blowout) regime, with $n_{b}>n_{0}$, can exceed the nonrelativistic wave-breaking limit, it is necessary to excite the plasma wave with a short pulse driving electron beam, in order that the plasma electrons not move to shield the beam charge during beam passage. This requirement can be stated as $k_{p} \sigma_{z} \leq 2$, where $\sigma_{z}$ is the rms length of the driving electron bunch. The mean electric field experienced by the driving beam can be estimated by viewing the wakefield generation as a generalized coherent Čerenkov interaction [4,17], to give $e E_{z, \text { dec }} \cong e^{2} N_{b} k_{p}^{2} / 2$. The accelerating wakefield amplitude behind the driving beam is typically $E_{z \text {,acc }} \cong$ $2 E_{z, \text { dec }}$, so we may write

$$
\frac{e E_{z, \mathrm{acc}}}{e E_{W B}} \cong \frac{e^{2} N_{b} k_{p}^{2}}{m_{e} c^{2} k_{p}}=r_{e} N_{b} k_{p},
$$

with $N_{b}$ equal to the bunch population. This ratio is not necessarily greater than unity for all conditions of blowout-it is, in fact, approximately 0.1 in the experiments described in this paper.

This situation, in which the beam is denser than the plasma, but does not drive longitudinal wakes near to the wave-breaking amplitude, is encountered when the beam radius or length is too small, so that even though the beam is dense enough to achieve blowout, it does not have enough charge to drive large wake fields. This is quantified as follows: for a bi-Gaussian beam distribution with dimensions $\sigma_{z}=\alpha_{z} / k_{p}$ and $\sigma_{r}=\alpha_{r} / k_{p}$, with the requirements $\alpha_{r}<1$ (the plasma electron motion is strongly radial), $\alpha_{z} \leq 2$ (the excitation is near to the maximum attained with an instantaneous impulse), the ratio of beam to plasma density is

$$
\frac{n_{b}}{n_{0}} \cong \frac{N_{b} k_{p}^{3}}{(2 \pi)^{3 / 2} \alpha_{r}^{2} \alpha_{z} n_{0}}=\frac{2 r_{e} N_{b} k_{p}}{(2 \pi)^{1 / 2} \alpha_{r}^{2} \alpha_{z}} .
$$

Thus we have, combining Eqs. (2) and (3),

$$
\frac{e E_{z, \mathrm{acc}}}{e E_{W B}} \cong \sqrt{\frac{\pi}{2}} \alpha_{r}^{2} \alpha_{z} \frac{n_{b}}{n_{0}},
$$

and, even for ratios of the beam/plasma density larger than unity, the accelerating fields may fall well short of wave breaking if the beam is narrow $\left(\alpha_{r} \ll 1\right)$ or short $\left(\alpha_{z} \ll 1\right)$. As the maximum wake fields will be obtained when the plasma is chosen as dense as possible while still allowing impulsive excitation of the wave, we inevitably choose $k_{p} \cong 2 / \sigma_{z}\left(\alpha_{z} \cong 2\right)$, and thus the optimized (i.e., the wakes at the most favorable plasma density) wake 
amplitude is a strong function of the bunch length,

$$
e E_{z, \mathrm{acc}} \cong \frac{2 e^{2} N_{b}}{\sigma_{z}^{2}}
$$

With this constraint on the optimum choice of $k_{p}$, the value of $\alpha_{r}$ is not chosen independently, but for a matched beam is a function of beam emittance and energy, as we now discuss.

All of the wake field characteristics examined so far concern the attributes of the fields in the rarefaction region, where the accelerating beam must be located in order to be propagated without transverse phase space dilution. The transverse wake fields for the drive beam, however, are not so uniform, because the plasma must take a finite time to respond to the beam. Because of this, the leading edge of the beam expands as if it were (ignoring small Coulomb scattering effects) in free space. On the other hand, the main body of the drive beam can be stably matched to the uniform focusing of the electron-rarefied ion channel. If the beam density is high enough, and the emittance is low, then the erosion of the beam head is not an important effect in our experimental parameter regime $[18,19]$.

The question of whether the beam can self-consistently propagate in the plasma without excessive transverse expansion has been explored in great detail analytically, computationally [14], and, as discussed in the next section, experimentally [5]. The work of Ref. [18] presents an analytical model of how rarefaction must proceed, assuming the entire beam is in fact matched (in the betatron sense, with no envelope oscillations) to the ion channel focusing. We review the relevant results from Ref. [19] here. Given the constraint $\alpha_{z} \leq 2$, the condition that the plasma electrons be rarefied by radial expulsion due to beam space-charge fields before the arrival of the tail end of the drive beam yields the constraint on the beam parameters, $N_{b} \geq 9 \varepsilon_{n} / \sqrt{4 \pi \gamma} r_{e}$, which is, interestingly, equivalent to $n_{b} \cong n_{0}$, with $\alpha_{z} \cong 2$. This condition can be satisfied by a high quality rf photoinjector [20-22]. It is, however, a bit of an optimistic model; beam-head erosion due to the fact that the beam head feels less focusing during the blowout process than the tail is not self-consistently taken into account. Computational studies of the effects of beam-head erosion, performed with (i) Maxwell-Vlasov beam/plasma electron fluid computational mode, (ii) superparticle beam/plasma electron fluid computational model, and (iii) a fully selfconsistent particle-in-cell code, all indicate that one needs approximately a factor of 2.5 larger charge to achieve rarefaction behind the drive beam [15],

$$
N_{b} \geq \frac{6 \varepsilon_{n}}{\sqrt{\gamma} r_{e}} \cong \frac{\varepsilon_{n}(\mathrm{~mm} \mathrm{mrad})}{\sqrt{E}(\mathrm{MeV})} \cdot 1.5 \times 10^{9} .
$$

The results of the study in Ref. [18] are quite relevant to the experiments we describe below. In particular, the inequality given in Eq. (6) indicates that the Argonne Wakefield Accelerator (AWA), which was not originally designed for low emittance operation, can drive a plasma wake field in the blowout regime, but with a very small margin of error.

\section{PRELIMINARY EXPERIMENTAL WORK AT ANL}

A number of experimental measurements of the PWFA in the overdense $\left(n_{0} \gg n_{b}\right)$ regime were carried out at ANL using the Advanced Accelerator Test Facility (AATF) setup [23]. These include first observation of plasma wakefield acceleration and focusing [24], selffocused beam propagation [25], and excitation of nonlinear plasma wake fields [26]. After the advantages of operation in the underdense, blowout regime were realized, an experiment designed to observe $\mathrm{GV} / \mathrm{m}$ accelerating wakes in this regime at the AWA photoinjector [3,27] facility was begun. The experimental goals were based on assumption of an electron beam with charge of $100 \mathrm{nC}$, $\sigma_{z}=0.75$, and a normalized rms emittance $\varepsilon_{n}$ less than a few hundred $\mathrm{mm}$ mrad. The bunch length achieved at the AWA has to this point never approached this value, however, and has been observed to be proportional to charge above approximately 10-15 nC [5,19,28], below which it approaches the value $\sigma_{z}=2.5 \mathrm{~mm}$. The scaling of Eq. (5) indicates, therefore, that the optimum wake fields would be observed at the lowest charge where this pulse lengthening effect asserts itself. In this case, according to Eq. (5), operation at $15 \mathrm{nC}$ derates the expected wakefield amplitude from the original design value $(1.9 \mathrm{GV} / \mathrm{m})$, by a factor of 0.03 , or $60 \mathrm{MV} / \mathrm{m}$. As we shall see below, this simple prediction is consistent with what has been observed.

The initial attempts at measuring wake fields in the blowout regime occurred in 1995, and were conducted with the experimental setup, shown in Fig. 3, at the AWA. The electron beam in this set of measurements had a mean energy of $14.5 \mathrm{MeV}$, with charge of 13-17 $\mathrm{nC}$, and no rigorous estimate of the emittance available at the time. This beam was focused into the plasma at near the matched beta function

$$
\beta_{\mathrm{eq}}=\sqrt{\gamma / 2 \pi r_{e} n_{0}}
$$

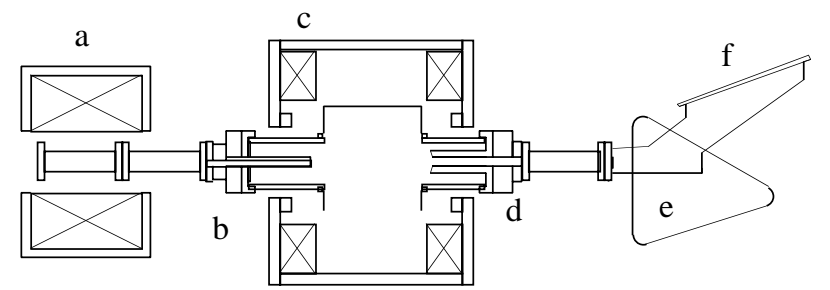

FIG. 3. Experimental setup including (a) focusing solenoid, (b) cathode assembly, (c) plasma confinement solenoid, (d) anode assembly, (e) bend magnet, and (f) phosphor screen. 
by use of the upstream solenoid. In the measurements of this run, the plasma density was $n_{0}=2.2 \times 10^{13} \mathrm{~cm}^{-3}$, obtained by use of the magnetically confined, hollow cathode arc source, featuring gas feed in the annular off-axis region formed by dual tantalum cathode tubes $[5,21,22]$. The plasma length is set in this device by the interelectrode distance, which in these experiments was $12 \mathrm{~cm}$. The plasma density in this device is mapped out with electrostatic probes, which have been calibrated by use of a $140 \mathrm{GHz}$ microwave interferometer [29].

While the AWA facility now has a $5 \mathrm{MeV}$ witness beam derived from a separate photoinjector, this lowenergy witness beam was not yet commissioned in 1995, and so could not be considered for use. In addition, the dramatic focusing provided by the matching solenoid would cause the beam dynamics for a lower energy beam to be very difficult to simultaneously match to the plasma focusing. Because of this, we employed a scheme in which a witness beam was generated in the main AWA photoinjector along with the drive pulse. This was accomplished by removing a central disk region of a cathodedrive laser transport mirror, and providing a sliding delay ( $\sim 20 \%$ of the full laser pulse) of the photons in this disk by changing the longitudinal position of a small mirror behind the mirror with the missing disk. This allowed production of a witness beam, which had a similar energy and density, and thus beam-plasma frequency, as the drive beam. This is a necessary condition for stable propagation of both beams in the strong focusing of a spacecharge dominated beam transport system [30,31]. This two-beam system could produce drive-witness delays as long as $70 \mathrm{psec}$, corresponding to laser path differences of about $3 \mathrm{~cm}$. Figure 4 shows a streak camera trace resulting from light emitted inside of a $1 \mathrm{~mm}$ thick fused silica Cerenkov radiator placed in the path of the beam (inclined $11^{\circ}$ to the normal), with the light directed to a Hamamatsu C1587 temporal disperser. Since the drive-witness

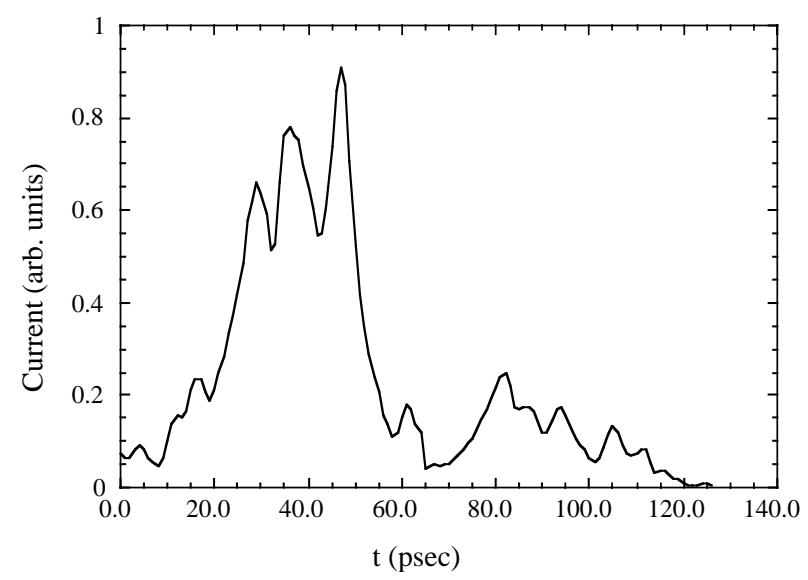

FIG. 4. Streak camera trace of beam-derived Čerenkov radiation, showing $14.5 \mathrm{MeV}$ drive and witness beam profiles generated simultaneously in the AWA photoinjector. delay time is dependent on the amount of compression the photoelectrons undergo during rf acceleration, the streak camera measurement was necessary to calibrate this delay time for a specific set of conditions (e.g., beam charge and size, acceleration field, and injection phase).

After we had developed the technique of witness beam generation, systematic measurements were made with the experimental setup shown in Fig. 3. In these experiments, the drive and witness beams combined had $Q=13-17$, and could be focused to a $\sigma_{r}=450 \mu \mathrm{m}$ spot at the plasma entrance. To investigate the possibility of particles accelerated by the plasma wave, we recorded images of the high-energy end of the spectrometer's phosphor screen. Figure 5 shows the intensity profiles for all possible combinations of switching on and off of witness beam and plasma. Each point on this plot is the result of several images from the camera, averaging the set of energies corresponding to a fixed intensity.

With no plasma present, the beam energy distributions are identical. This suggests that prior to entering the plasma, the witness beam's high-energy tail is at or below that of the drive beam and does not appear in the plots. Without a method to diagnose the witness beam alone, we could only conclude that the lower limit on the witness beam's gain in energy as a result of the beam-plasma interaction is $0.5 \mathrm{MeV}$. Note that at lowest intensity, the gain in energy of the tail is approximately twice as large again. Therefore the average acceleration field was at least as large as $4.1 \mathrm{MeV} / \mathrm{m}$ in this run.

When trying to compare these data with simulation results, we found that a $\sigma_{r}=450 \mu \mathrm{m}$ beam's core focuses to $180 \mu \mathrm{m} \mathrm{rms} \mathrm{radial} \mathrm{size} \mathrm{inside} \mathrm{of} \mathrm{the} \mathrm{plasma,}$ and then oscillates while staying below $330 \mu \mathrm{m}$. This implies that in places along the propagation the beam is 4 times denser than the plasma, locally satisfying

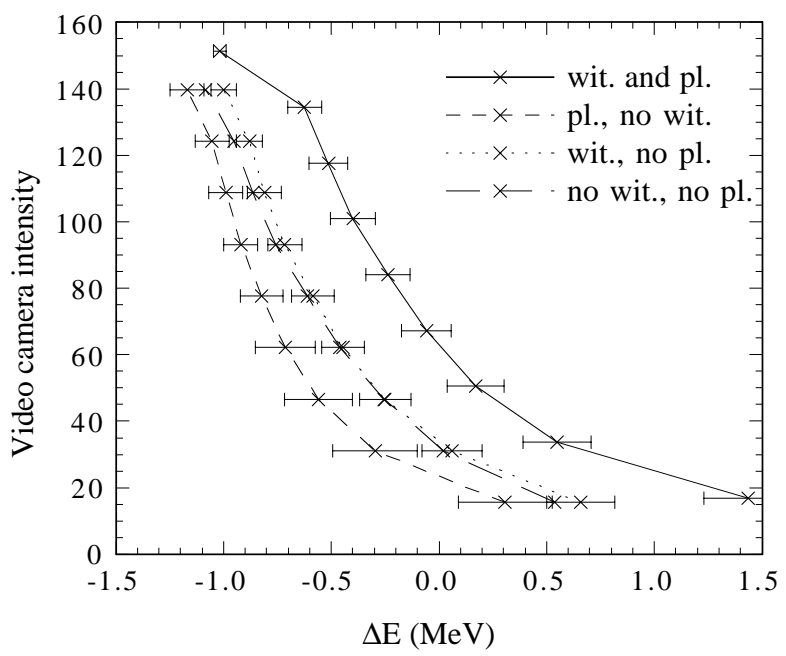

FIG. 5. Observation of the high-energy tail at spectrometer. In the legend, "wit." indicates witness beam and "pl." indicates plasma present. 
the underdense criterion. The witness beam in this experiment was positioned so far back that it overlapped with the second peak in the acceleration field. The simulation corresponding to this case resulted in the formation of wake fields at $20 \mathrm{MeV} / \mathrm{m}$ averaged over the propagation, assuming a $13 \mathrm{nC}$ drive beam, a number not in disagreement with measurements. Major possible sources of disagreement between the experiment and the simulations are observed nonaxisymmetries in the beam distribution due to cathode nonuniformity, space charge, and transverse wakefield effects. These effects were observed in the next round of acceleration experiments.

Although these experiments yielded some advances in our experimental technique, notably the novel method of witness beam generation, there were several unsatisfactory aspects of the measurements. The first is that the beam, from these measurements, was known to be roughly as dense as the plasma at the beginning of the plasma column, and suspected to be much denser inside of the column. However, no actual experimental information was known about its propagation within the plasma. Also, the spread of the witness beam in time (see Fig. 4) was not much smaller than the wavelength, and so the time resolution of the measurement was not appropriately small. In addition, the resolution of the energy spectrometer given such a large transverse emittance beam was not very good. Because of these shortcomings, we undertook a number of actions to improve the experiment: we upgraded the upstream beam diagnostics, in order to establish our initial conditions better; we performed a detailed measurement of the matched beam propagation in the plasma, establishing unequivocally that the beam was denser than the plasma; and we upgraded the energy measurement system with a new spectrometer, as well as a slit-collimation introduced in the propagation experiments.

The results of the matched beam propagation experiments are described in great detail in Ref. [6], but the methods and results of these experiments are of high importance for understanding of the acceleration results we present in the next section, and so we review them here. The purpose of the acceleration experiments was to reliably measure beam charge, bunch length, and the transverse beam profiles at both the entrance and the exit of the plasma column, in order to create and diagnose the selfguided conditions necessary for long range acceleration in the blowout regime. These experiments were the first to test underdense plasma lens action [32] in the short-pulse (wakefield acceleration) regime $[18,19]$.

The experimental setup for these measurements is shown in Fig. 6. The upstream region of the beamline between the AWA photoinjector and the plasma was instrumented for charge, energy, emittance, and beam profile measurements, in order to establish the initial conditions on the beam state, even in the presence of relatively large shot-to-shot fluctuations in the beam charge. The beam charge and profile characteristics directly at the plasma

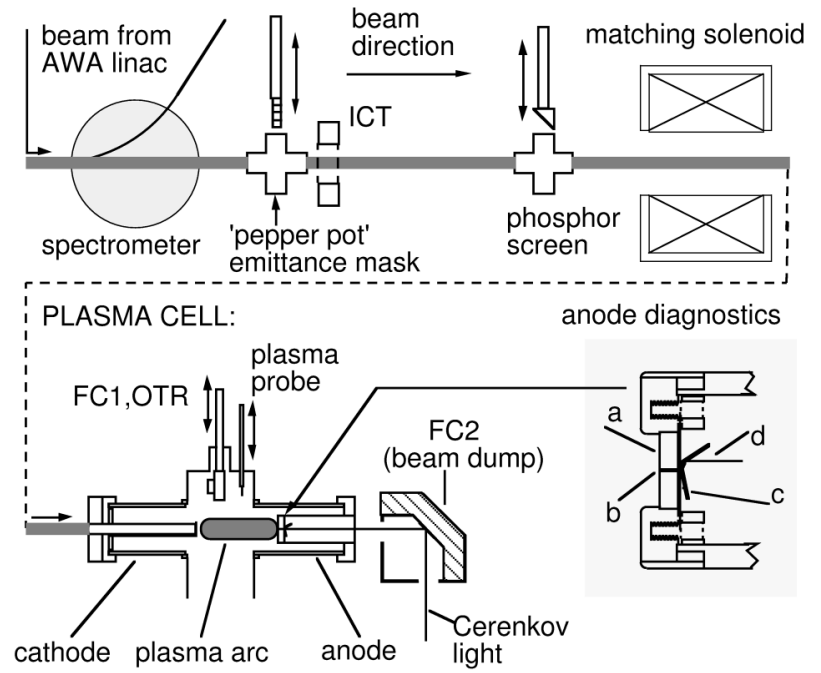

FIG. 6. Diagnostics beamline and plasma cell (shown without the plasma radial confinement solenoid). The anode diagnostics include (a) tungsten collimator with (b) $1 \mathrm{~mm}$ wide slit, (c) $500 \mu \mathrm{m}$ thick quartz Čerenkov plate, and (d) mirror and outgoing light.

entrance are established by use of the insertable Faraday cup FC1 and the optical transition radiation (OTR) mirror inside of the plasma chamber, respectively. The downstream diagnostics assembly, located at the plasma exit, begins with a tungsten collimator having a $1 \mathrm{~mm}$ wide slit aperture in it, followed by a $500 \mu \mathrm{m}$ thick quartz Čerenkov plate, and a mirror to relay the Čerenkov light to either a CCD camera or a streak camera. The Čerenkov light allowed for both time-integrated and time-resolved imaging of the beam profile at the plasma exit. The slit assembly allowed for an independent check on the focusing, by giving a fractional charge passed signal obtained from comparison of the upstream nondestructive integrating current transformer (ICT) measurement, and the charge collected in the beam dump Faraday cup (FC2). The combination of the two diagnostics gave a powerful veto on shots that were off-slit center, or too asymmetric.

The analysis of the data obtained in this experiment agreed well with the comparison to simulations, and verified the general conclusions of the computational and theoretical analysis of the beam propagation characteristics found in Refs. [18] and [19]. In summary, it was found that the $14.5 \mathrm{MeV}, Q=14 \mathrm{nC}, \varepsilon_{n} \cong \gamma \varepsilon \cong$ $150 \mathrm{~mm}$ mrad beam could be focused to an initial spot at the plasma entrance OTR screen of initial spot of $\sigma_{r} \cong 280 \mu \mathrm{m}$, corresponding to an initial beam function of the waist of $\beta_{i} \cong 1.5 \mathrm{~cm}$. In the absence of ionderived plasma focusing, this beam expands by a factor of 3 by the time it encounters the slit, and very little charge is passed to the diagnostics. When the plasma $\left(n_{0}=1.15 \times 10^{13} \mathrm{~cm}^{-3}\right)$ is turned on, the body of the beam propagates in its self-formed ion channel, with equilibrium beta function $\beta_{\text {eq }} \cong 1.25 \mathrm{~cm}$, which is very close 
to a matched case, considering the effects of the initial ramping up of the plasma density [15]. Most of the charge passes through the slit collimation system at the plasma exit, with the time-integrated and time-resolved transverse profiles giving a detailed picture of the beam distribution at the plasma exit. The beam, with measured temporal width FWHM of $25 \mathrm{psec}\left(k_{p} \sigma_{z}=1.9\right)$, was determined to propagate with peak density of at least $n_{b} / n_{0} \cong 2.5$. The agreement of the time-resolved beam profile measurements with simulation was very good, as the beam showed the characteristic "trumpet" shape of an expanded beam head, with the beam body well matched to the focusing, essentially not expanding over a distance greater than 8 times the initial beta function. The success of this experiment, at both creating and diagnosing the condition of blowout in the beam-plasma interaction, allowed us to proceed to the next round of acceleration experiments.

\section{ACCELERATION AND DECELERATION IN THE BLOWOUT REGIME}

The latest set of experiments was performed with the upstream beam diagnostics, matching optics and diagnostics, and plasma source in essentially the same configuration as used in the blowout propagation experiments described above. After the downstream end of the plasma, the tungsten collimator slit assembly remained, while the beam diagnostics were converted from transverse to energy measurements, and the beam charge measurement was changed to a nondestructive ICT. The energy spectrum was measured in this case by use of a high-resolution (as compared with the device shown in Fig. 5) magnetic dipole spectrometer constructed expressly for this experiment. A vertically focusing quadrupole magnet upstream of the spectrometer allowed all of the beam charge to be transported through the dipole magnet gap, and to give an optimized image of highest resolution, at the focal plane of the spectrometer.

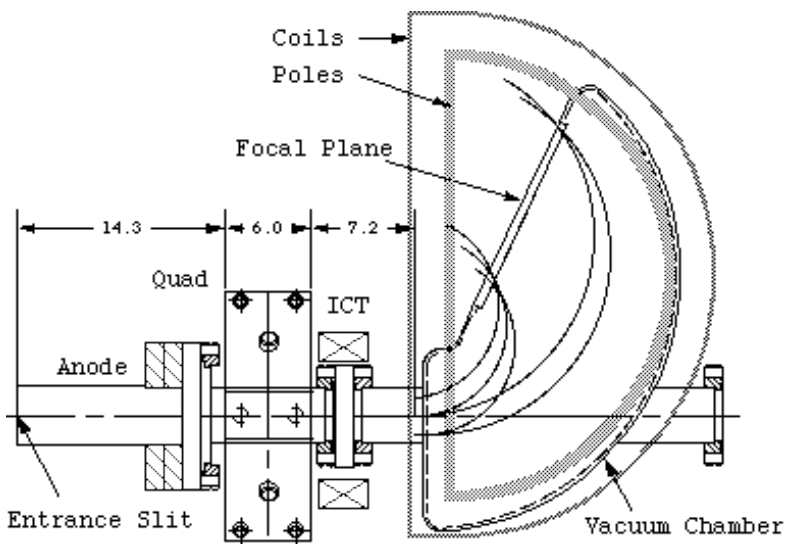

FIG. 7. Downstream beam diagnostics, with slit collimation in plasma arc anode, quadrupole lens, and high-resolution magnetic spectrometer with example trajectories.
The collimating slit assembly serves the same primary purpose in this experiment as in the propagation experiment, that of ensuring that one has tuned for the best transverse focusing match to the plasma, as measured by the fractional transmission through the slits. It also serves as a filter to the particles accepted into the spectrometer, as only particles with small horizontal offset, and therefore within or near the plasma-electron rarefied beam channel will pass the slits. As the slits provide a horizontally narrow "source," the initial conditions for the quadrupole/ spectrometer beam optics are well defined, the tuning of the spectrometer optics is made more straightforward, and the ultimate spectral resolution of the device improved. The slits also allow the dimension along the slit (vertical) to be used for observation of transverse information, such as evidence for the electron hose instability [33], while the direction normal to the slit is used for energy dispersion. This is analogous to setup for the propagation experiments, in which the dimension parallel to the slits was used for spatial profile information, with the direction normal to the slits reserved for temporal dispersion.

The additional experience in operation of the AWA, and to matching of the beam to the induced plasma focusing, over the previous runs was evident in the achieved experimental parameters, which are summarized in Table I. The beam charge, as measured in the plasma chamber at $\mathrm{FC} 1$, was raised in this experiment to a mean value of $18 \mathrm{nC}$ (again with large rms fluctuations of $\pm 5 \mathrm{nC}$ ), while the pulse length was shortened a small amount to a mean FWHM value of 20-24 psec. This beam, optimized for energy exchange with the plasma rather than propagation as in Ref. [6], had larger normalized emittance $\varepsilon_{n} \cong$ $180 \pm 30 \mathrm{~mm}$ mrad than before and was again slightly mismatched to the plasma focusing. The shorter bunch length allowed a slightly larger plasma density of $n_{0} \cong$ $1.3 \times 10^{13} \mathrm{~cm}^{-3}\left(k_{p} \sigma_{z}=1.75-2\right)$ to be used in this round of experiments. The mean ratio of the beam-toplasma density for the average case is thus approximately $n_{b} / n_{0} \cong 3$ at the beginning of the plasma, giving very underdense conditions. The fractional beam transmission past the plasma exit slits was not as high (less than 0.4) in this round of experiments as in the propagation experiments (less than 0.7). This result is consistent with the simulations of the experiment, which are described below. It is due to the effects of running with a larger beam emittance and smaller $k_{p} \sigma_{z}$ in this experiment,

TABLE I. Experimental parameters for blowout regime plasma wakefield acceleration experiment at the AWA.

\begin{tabular}{lc}
\hline \hline Beam energy & $15.6 \mathrm{MeV}$ \\
Beam charge $(Q)$ & $18 \pm 5 \mathrm{nC}$ \\
Pulse length $(\mathrm{FWHM})$ & $20-24 \mathrm{psec}$ \\
Initial rms transverse size $\left(\sigma_{r}\right)$ & $250 \mu \mathrm{m}$ \\
Mean peak beam density $\left(n_{b}\right)$ & $4.0 \times 10^{13} \mathrm{~cm}^{-3}$ \\
Plasma electron density $\left(n_{0}\right)$ & $1.3 \times 10^{13} \mathrm{~cm}^{-3}$ \\
\hline \hline
\end{tabular}


both of which enhance beam-head erosion, but were a necessary result of our experimental maximization of observed beam acceleration. The use of a larger peak current beam allowed higher energy gain to be observed, but with its higher emittance had the unfortunate attribute of enhanced beam-head erosion, as is discussed below.

Because the witness beam which could be produced in the main AWA photoinjector was not notably shorter than the drive beam, as seen in Fig. 4, and furthermore was quite difficult to align to the drive beam (to the degree that both could be successfully propagated through the slits), these experiments were performed with only the drive beam tail used to measure acceleration. It should be noted that this method is also to be employed in the plasma wakefield acceleration experiment E-157 at the SLAC Final Focus Test Beam Facility [13]. The full spectrum of the drive beam was then observed in the focal plane of the spectrometer, producing momentum spectra along the dispersive direction, as seen in Figs. 8 and 9 for cases with and without plasma present. The momentum spectrometer video data, as well as the ICT signals for each shot were recorded, and a large number of shots at a given set of beam and plasma conditions were taken for this experiment.

Without plasma present, the beam's minimum rms momentum spread of $1 \%$ [Fig. 8(a)] was smaller than measured with the previous spectrometer (with no slits), indicating that the resolution of the momentum (or, alternatively, energy) measurement system was improved with a better defined source provided by slit collimation.

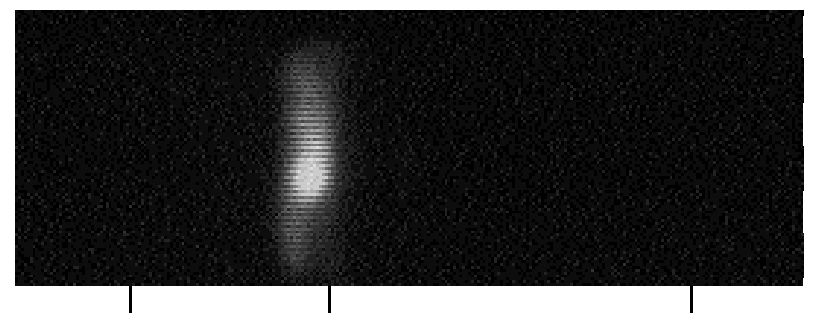
$14.0 \mathrm{MeV}$
$15.6 \mathrm{MeV}$
(a)
$18.6 \mathrm{MeV}$

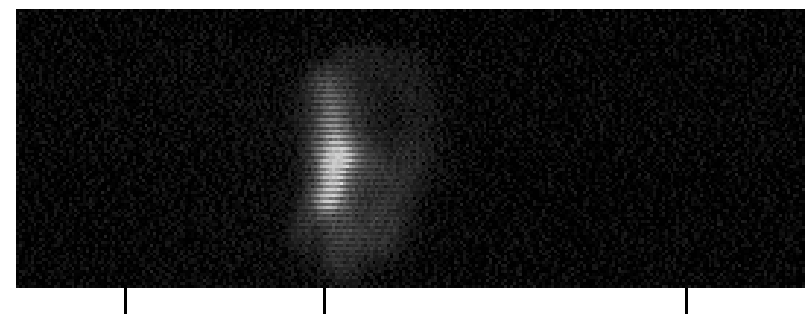

$14.0 \mathrm{MeV} \quad 15.6 \mathrm{MeV}$

(b)

$18.6 \mathrm{MeV}$

FIG. 8. Video image made by beam, with no plasma present, striking phosphor of focal plane in momentum analyzing spectrometer, for (a) typical narrow spectrum case and (b) larger momentum spectrum due to injection phase fluctuation. Note the transverse (vertical dimension) structure of the image in this case.
Injection timing jitter of the photocathode drive laser with respect to the rf wave, which is estimated at $7 \mathrm{psec}$ rms $\left(3.1^{\circ}\right)$ caused the beam to have a momentum centroid jitter of $0.9 \%$. A shot with maximal injection centroid error, of course, has enhanced energy spread. This is illustrated in Fig. 8(b), where the momentum spectrum is larger because of the correlated energy spread due to injection timing error. This correlation allows a structure to be observed in the nondispersive dimension. This type of structure is due to strongly fluctuating intensity nonuniformities within the laser pulse envelope. These intensity nonuniformities, which differ from shot to shot [34], produce large effects in the energy measurements in some shots.

These effects are displayed in Fig. 9, which shows (i) a well-behaved shot, with little structure in the nondispersive plane and large deceleration/acceleration observed, and (ii) a shot with considerable filamentary structure and less acceleration. It was clear that the more poorly behaved shots had structure in the dimension along the slit, which would lead the driving of a less symmetric, poorer quality wave, deflection of the beam tail, and a smaller region that can optimally accelerate electrons. These effects lead to less observed total acceleration in the filamentary cases. As the drive beam itself provides the accelerating particles, the observed accelerated spectrum is also a function of the filamentary nature of this beam. Note also the existence of
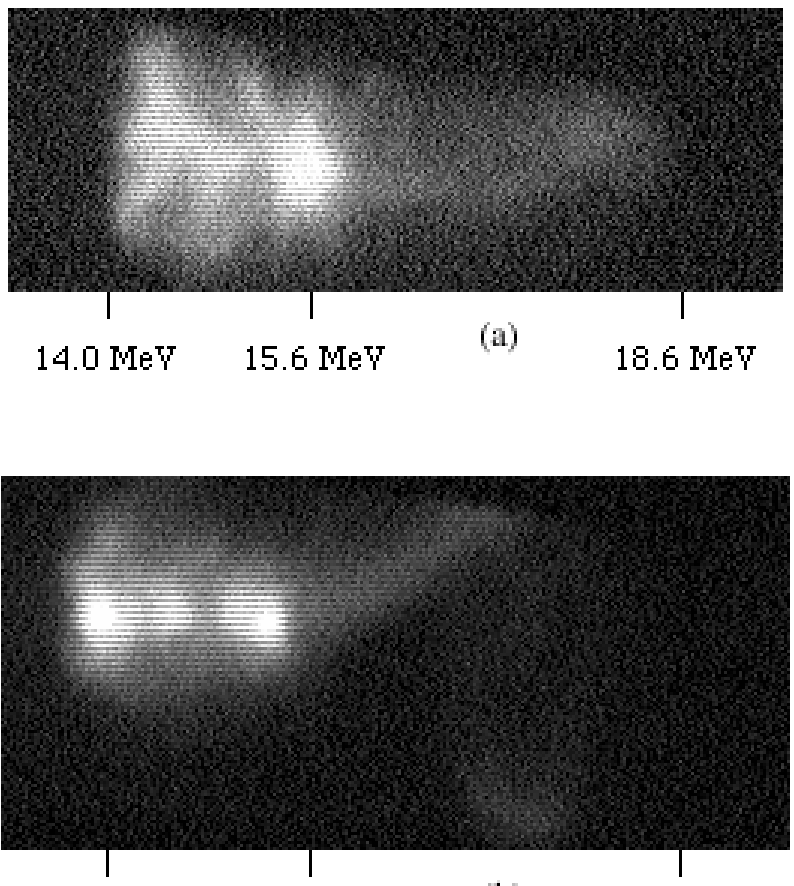

$14.0 \mathrm{MeV} \quad 15.6 \mathrm{MeV}$

(b)

$18.6 \mathrm{MeV}$

FIG. 9. Video image made by beam, with plasma present, in momentum analyzing spectrometer, for (a) a good acceleration case $(Q=19 \mathrm{nC})$ and (b) a case where the tail is deflected, and less acceleration is observed. Note hot spots centered at distinct energies. 
hot spots, centered at distinct energies, in the spectrometer focal distribution in both Figs. 9(a) and 9(b), which is further evidence that the beam distribution is not smooth, but filamentary. This filamentation of the beam in energy may indicate that the initial longitudinal distribution is not smooth, but has notable structure. This type of structure is not readily observable in picosecond-resolution streak camera images, however, due to the inherent noisiness of this type of measurement.

It would be interesting to be able to attribute the transverse filamentation of the beam in the spectrometer to electron hose instability $[19,33]$. This cannot be done with confidence from these data, however, as while the observed maximum transverse offset in the spectrometer is large for filamentary shots, it is not enough larger than the maximum offset for well-behaved shots, as illustrated by Figs. 9(a) and 9(b), to justify the claim of instability onset. In fact, it may be possible that the dramatic structures observed in shots like Fig. 9(b) are due in large part to the simple expansion of the momentum spectrum in combination with the plasma focusing transporting more electrons through the slit. These effects certainly allow the filamentary nature of the beam in configuration and phase space to be more clearly observed.

The energy spectra for the shots observed at the optimum beam and plasma conditions given in Table I have been further analyzed. Three quantities have been extracted from analysis of these spectra: (i) the peak in the spectrum, which should be due to the electrons in the region near the beam head that are well guided but not strongly decelerated, (ii) the lowest resolvable energy, and (iii) the highest resolvable energy. These quantities have been plotted in Fig. 10 as a function of shot charge reported by the upstream ICT. While there was a large spread in maximum observed deceleration and acceleration at a given charge due to the intensity nonuniformities, the momentum of the spectrum peak had small fluctuations and, further, had a very small negative linear depen-

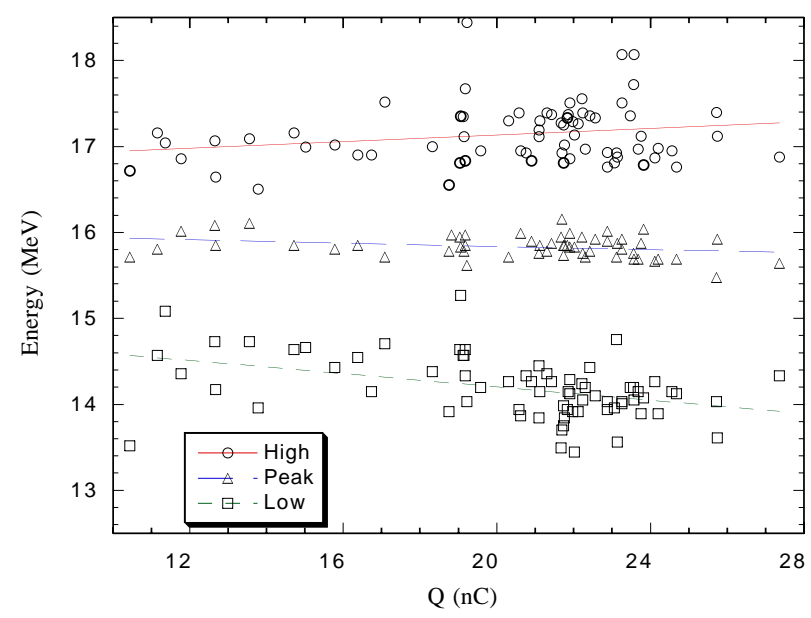

FIG. 10. Minimum, maximum, and peak intensity of measured energy spectra, as a function of charge $Q$. dence on charge. The maximum observed deceleration, which arises from the beam core, had smaller fluctuations at a given charge than the fluctuations in maximum acceleration, and a strong negative linear charge dependence. On the other hand, the linear charge dependence of the maximum observed acceleration was positive, but weaker than the charge dependence of the maximum observed deceleration, with the large fluctuations due to the facts that smaller numbers of electrons are available in the tail to be accelerated and these electrons can be easily steered away from the maximum acceleration region.

The spectrum for the well-behaved shot of Fig. 9(a) is displayed in Fig. 11, along with a simulated spectrum obtained from running the hybrid beam superparticle/ plasma fluid code [15,18] based on NOVO [7]. The simulated particles were loaded initially at the entrance of a uniform plasma (the plasma density is uniform to better than $10 \%$ over the interelectrode distance [29]) with a thermal, uncorrelated distribution in all phase planes. Explicitly, this means that Gaussian distributions with rms spreads corresponding to measured values in all Cartesian coordinate and momentum dimensions were launched at the plasma entrance, with no correlation at this point between any of the phase space dimensions. This same type of distribution, although clearly not in detailed agreement with the actual highly correlated beam obtained from the AWA photoinjector, was used in the computational analysis of the propagation experiments [6]. In these previous experiments, good agreement between the data and simulation was obtained, with a notable exception being that the erosion of the beam head is actually overestimated in the computations. This exception was due to the fact that the emittance at a given longitudinal slice of the beam is smaller than the total projected emittance [20,31].

The comparison between simulation and experiment in the present case is shown in Fig. 11, which displays the experimental energy spectra for the case of Fig. 9(a)

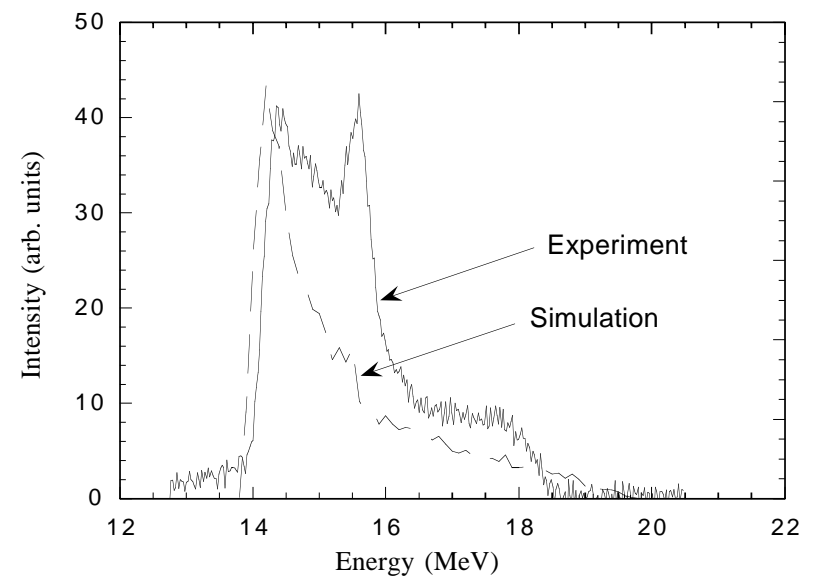

FIG. 11. Measured energy spectrum from the case of Fig. 9(a), compared to simulated (with hybrid superparticle/ plasma fluid code) spectrum. 
and a computer simulation, which included the final slit collimation. As the computational model assumes a perfectly symmetric, thermalized bi-Gaussian beam, we adjusted the simulation parameters within experimental uncertainty to give us the best spectral fit. In the case shown, we used the following parameters: $Q=17 \mathrm{nC}$, $\varepsilon_{n}=200 \mathrm{~mm} \mathrm{mrad}$, FWHM pulse length of $24 \mathrm{psec}$, an initial energy of $15.6 \mathrm{MeV}$, and plasma density of $n_{0}=1.25 \times 10^{13} \mathrm{~cm}^{-3}$. The simulation is in fairly good agreement with the experiment, with the experimental and simulated spectra displaying the same qualitative signatures in the deceleration and acceleration regions, as well as good quantitative agreement on the actual placement of the decelerated and accelerated distribution end points. The observed peak intensity of the spectrum near the initial energy is not well pronounced in the case of the simulation. This is due to the fact, known from the propagation experiments, that the beam-head region, which is weakly decelerated, guides better in experiment than in simulation, due to the slice emittance effect discussed above, as well as in Ref. [6]. Even with the lack of detailed agreement in the measured and simulated spectral peaks, the acceleration and deceleration end points are found to agree well.

The simulations give some further insight into the mechanisms responsible for the production of the spectral shapes given in Fig. 11. In particular, it is observed that beam-head erosion plays a large role in this type of experiment, which employs a relatively large emittance beam. Erosion of the beam head produces two effects: The first is that the beam-head expansion lowers the coupling of the beam to the plasma, producing a smaller amplitude wake. The second is that the wake, being produced effectively by regions which progress backwards in the beam frame, suffers a phase shift. Both of these effects give rise to a measured maximum average acceleration, which in the case of Figs. 9(a) and 11 is $25 \mathrm{MeV} / \mathrm{m}$, which is smaller than the peak maximum acceleration gradient in the wave. These effects are illustrated in Fig. 12, which shows the simulated longitudinal wake field near the plasma entrance $(z=1 \mathrm{~cm})$ and again near the plasma exit $(z=12 \mathrm{~cm})$. The peak accelerating field is degraded from $\left|e E_{z}\right|=0.18 m_{e} c \omega_{p}=62 \mathrm{MeV} / \mathrm{m}$ to $49 \mathrm{MeV} / \mathrm{m}$. Note though, that an electron initially accelerating on-axis at the wave peak for $z=1 \mathrm{~cm}$ suffers a degradation of acceleration rate from 62 to $35 \mathrm{MeV} / \mathrm{m}$ at $z=12 \mathrm{~cm}$. This effect partially explains the discrepancy between the peak accelerating field created in the plasma and the maximum average acceleration, a discrepancy which is nearly the same as in the simulation. The additional uncertainty associated with determining the population and initial energy of the longitudinal tail of the non-Gaussian AWA beam, as well as the difficulty in measuring the end point of the energy distribution in a noisy accelerator enclosure, may provide other mechanisms for explaining the observed maximum acceleration.

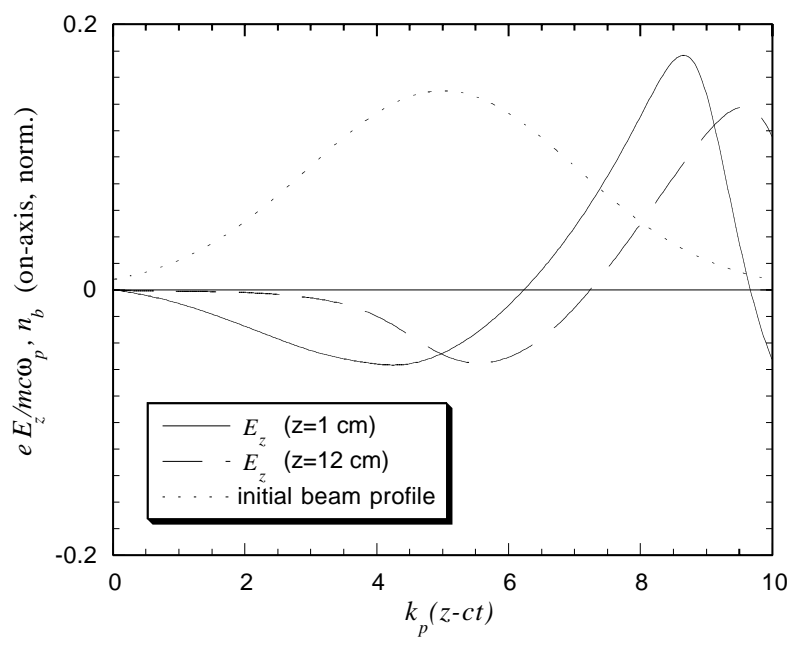

FIG. 12. The initial on-axis beam profile, as well as longitudinal wake field at the beginning of the plasma $(z=1 \mathrm{~cm})$ and end of the plasma $(z=12 \mathrm{~cm})$, from simulation. The wakefield amplitude and phase changes are due to beam-head erosion.

\section{CONCLUSIONS}

This round of experiments at the AWA facility gave the first measurements of plasma wakefield acceleration in the blowout regime. These measurements were made possible by first establishing methods of achieving a verifiable underdense plasma condition in a previous round of experiments [6]. The observed maximum acceleration gradient was $25 \mathrm{MeV} / \mathrm{m}$, corresponding to a peak field in the simulations presented of over $60 \mathrm{MeV} / \mathrm{m}$. While these measurements were consistent with the predictions of theory and simulation, they were difficult and fell short of design. The difficulties in these experiments were mainly derived from the beam quality - the emittance was high, the beam was filamentary, with large variations in charge and profile, and, most importantly, it was longer than originally expected. At present, the AWA rf gun is under redesign and will be replaced [35]. The next gun should produce much shorter, high charge bunches more suitable for driving large amplitude wake fields in the blowout regime.

The next round of measurements we currently plan is to take place at the Fermilab Test Facility $[14,34]$ rf photoinjector. This facility has several advantageous attributes, including a high quantum efficiency cesium telluride cathode, which can produce more uniform emission than the metallic cathodes used in this experiment, emittance compensated optics, and, most critically, a bunch compressor. With this source, we expect to be able to produce $19 \mathrm{MeV}, 14 \mathrm{nC}$ bunches, with normalized emittance of $\varepsilon_{n} \cong 80 \mathrm{~mm} \mathrm{mrad}$, and rms pulse length $\sigma_{z} \cong 0.4 \mathrm{~mm}$. With a plasma source of density $n_{0} \cong 10^{14} \mathrm{~cm}^{-3}$, this beam is predicted [14] to produce peak accelerating fields in excess of $1 \mathrm{GeV} / \mathrm{m}$ in the blowout regime. With a relatively low emittance and high current, the beam-head 
erosion problem is much smaller in this planned experiment than in the one reported here.

Erosion and driving beam distortion are signatures of low-energy beam experiments, as they are driven by both the larger geometrical emittances and nontrivial energy loss incurred in this type of experiment. This should not be a problem in the upcoming E-157 experiment at Stanford [13], which runs with a beam energy of $30 \mathrm{GeV}$. It would, however, enter into the design considerations for a multi$\mathrm{GeV}$ plasma acceleration module for a possible future linear collider design [5]. In this case, the phase shifts associated with long range erosion could be compensated by slow longitudinal variation of the plasma density.

As a final thought we note that, while we achieved the simultaneous creation of both a witness and a drive beam in a single rf photoinjector, the witness beam was of limited experimental use. While this was partly true because the AWA gun was not optimized for this task, it also points to the challenge of creating beams which will allow not only cleaner measurements in experiments, but give good emittances and energy spreads at the exit of a plasma accelerator. The plasma accelerators we have discussed here are envisioned to have wavelengths of $1 \mathrm{~mm}$ or so, and thus the accelerating beams must be considerably shorter than $1 \mathrm{~mm}$ (pulses in the sub-100 fsec regime), and also phase locked to these high frequency waves. While there are a number of conventional suggestions for creating these types of beams with rf photoinjectors and compressors [5], another promising path appears to be the use of plasma waves themselves as the source of the injected particles [36]. As the next generation plasma wakefield experiments will need both improved driving and witness beams, this should be an area of active research in the coming years.

\section{ACKNOWLEDGMENTS}

The authors would like to thank R. Konecny, A. Murokh, and P. Schoessow for experimental aid and support. This work was supported by U.S. Department of Energy Grants No. DE-FG03-92ER40693 and No. W31-109-ENG-38.

[1] C.E. Clayton et al., Phys. Rev. Lett. 70, 37 (1993).

[2] K. Nakajima et al., Phys. Rev. Lett. 74, 4428 (1995).

[3] P. Chen, J. M. Dawson, R.W. Huff, and T. Katsouleas, Phys. Rev. Lett. 54, 693 (1985).

[4] J. B. Rosenzweig, B. Breizman, T. Katsouleas, and J.J. Su, Phys. Rev. A 44, R6189 (1991).

[5] J. B. Rosenzweig, N. Barov, A. Murokh, E. Colby, and P. Colestock, Nucl. Instrum. Methods Phys. Res., Sect. A 410, 532 (1998).

[6] N. Barov, M.E. Conde, W. Gai, and J.B. Rosenzweig, Phys. Rev. Lett. 80, 81 (1998).

[7] B.N. Breizmann, T. Tajima, D.L. Fisher, and P.Z. Chebotaev (unpublished).
[8] A. I. Akhiezer and R. V. Polovin, Sov. Phys. JETP 3, 696 (1956).

[9] J. M. Dawson, Phys. Rev. 113, 383 (1959).

[10] J. B. Rosenzweig, Phys. Rev. Lett. 58, 555 (1987).

[11] T. Katsouleas and W. Mori, Phys. Rev. Lett. 61, 90 (1988).

[12] J. B. Rosenzweig, Phys. Rev. A 38, 3634 (1988).

[13] R. Assman et al., Nucl. Instrum. Methods Phys. Res., Sect. A 410, 396 (1998).

[14] J. Rosenzweig, N. Barov, E. Colby, L. Serafini, N. Bigelow, A. Fry, M. Fitch, P. Colestock, and R. Noble, Fermilab Report No. P890, 1996.

[15] J. B. Rosenzweig, Nucl. Instrum. Methods Phys. Res., Sect. A 410, 335 (1998).

[16] B. Montague and W. Schnell, in Laser Acceleration of Particles, edited by C. Joshi and T. Katsouleas (AIP, New York, 1985), p. 303.

[17] J.D. Jackson, Classical Electrodynamics (Wiley, New York, 1975), 2nd ed., pp. 641-643.

[18] N. Barov and J.B. Rosenzweig, Phys. Rev. E 49, 4407 (1994).

[19] J. Krall and G. Joyce, Phys. Plasmas 2, 1326 (1995).

[20] X. Qiu et al., Phys. Rev. Lett. 76, 3723 (1996).

[21] J. B. Rosenzweig, S. Anderson, K. Bishofberger, X. Ding, A. Murokh, C. Pellegrini, H. Suk, A. Tremaine, C. Clayton, C. Joshi, K. Marsh, and P. Muggli, Nucl. Instrum. Methods Phys. Res., Sect. A 410, 437 (1998).

[22] M.E. Conde, W. Gai, R. Konecny, X. Li, J. Power, P. Schoessow, and N. Barov, Phys. Rev. ST Accel. Beams 1, 041302 (1998).

[23] H. Figueroa, W. Gai, R. Konecny, J. Norem, P. Schoessow, and J. Simpson, Phys. Rev. Lett. 60, 2144 (1988).

[24] J. B. Rosenzweig, D. B. Cline, B. Cole, H. Figueroa, W. Gai, R. Konecny, J. Norem, P. Schoessow, and J. Simpson, Phys. Rev. Lett. 61, 98 (1988).

[25] J. B. Rosenzweig, P. Schoessow, C. Ho, W. Gai, R. Konecny, S. Mtingwa, J. Norem, M. Rosing, and J. Simpson, Phys. Fluids B 2, 1376 (1990).

[26] J. B. Rosenzweig et al., Phys. Rev. A 39, 1586 (1989).

[27] P. V. Schoessow, in Proceedings of the 1997 Particle Accelerator Conference, Vancouver, Canada, edited by M. Craddock (IEEE, Piscataway, NJ, 1998), p. 639.

[28] N. Barov et al., in Proceedings of the 1995 Particle Accelerator Conference, Dallas, Texas (IEEE, Piscataway, NJ, 1996), p. 631.

[29] N. Barov, Ph.D. thesis, UCLA, 1998.

[30] J. B. Rosenzweig and E. Colby, Advanced Accelerator Concepts, edited by P. Schoessow (AIP, New York, 1995), p. 724.

[31] Luca Serafini and J. B. Rosenzweig, Phys. Rev. E 55, 7565 (1997).

[32] J. J. Su, T. Katsouleas, J. Dawson, and R. Fedele, Phys. Rev. A 41, 3321 (1990).

[33] D. Whittum, W. Sharp, S. S. Yu, M. Lampe, and G. Joyce, Phys. Rev. Lett. 67, 991 (1991).

[34] Eric Colby, Ph.D. thesis, UCLA, 1998.

[35] W. Gai, X. Li, M. Conde, J. Power, and P. Schoessow, Nucl. Instrum. Methods Phys. Res., Sect. A 410, 431 (1998).

[36] D. Umstadter et al., Phys. Rev. Lett. 76, 2073 (1996). 